\title{
Description of nucleation, growth, and coagulation processes in the modeling of debris formation after a nuclear burst
}

\section{Approved for public release.} Distribution is unlimited.

\section{OAK RIDGE NATIONAL LA




\section{DOCUMENT AVAILABILITY}

Reports produced after January 1, 1996, are generally available free via US Department of Energy (DOE) SciTech Connect.

Website: www.osti.gov/

Reports produced before January 1, 1996, may be purchased by members of the public from the following source:

National Technical Information Service

5285 Port Royal Road

Springfield, VA 22161

Telephone: 703-605-6000 (1-800-553-6847)

TDD: $703-487-4639$

Fax: 703-605-6900

E-mail: info@ntis.gov

Website: http://classic.ntis.gov/

Reports are available to DOE employees, DOE contractors, Energy Technology Data Exchange representatives, and International Nuclear Information System representatives from the following source:

Office of Scientific and Technical Information

PO Box 62

Oak Ridge, TN 37831

Telephone: 865-576-8401

Fax: 865-576-5728

E-mail: report@osti.gov

Website: http://www.osti.gov/contact.html

This report was prepared as an account of work sponsored by an agency of the United States Government. Neither the United States Government nor any agency thereof, nor any of their employees, makes any warranty, express or implied, or assumes any legal liability or responsibility for the accuracy, completeness, or usefulness of any information, apparatus, product, or process disclosed, or represents that its use would not infringe privately owned rights. Reference herein to any specific commercial product, process, or service by trade name, trademark, manufacturer, or otherwise, does not necessarily constitute or imply its endorsement, recommendation, or favoring by the United States Government or any agency thereof. The views and opinions of authors expressed herein do not necessarily state or reflect those of the United States Government or any agency thereof. 
Nuclear Nonproliferation Division

\title{
DESCRIPTION OF NUCLEATION, GROWTH, AND COAGULATION PROCESSES IN THE MODELING OF DEBRIS FORMATION AFTER A NUCLEAR BURST
}

\author{
Pablo Moresco
}

Date Published: January 2021

\author{
Prepared by \\ OAK RIDGE NATIONAL LABORATORY \\ Oak Ridge, TN 37831-6283 \\ managed by \\ UT-Battelle, LLC \\ for the \\ US DEPARTMENT OF ENERGY \\ under contract DE-AC05-00OR22725
}





\section{CONTENTS}

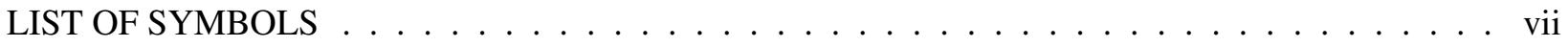

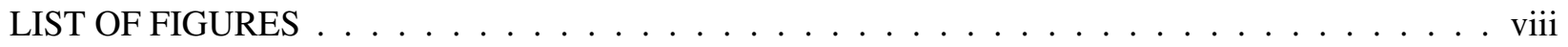

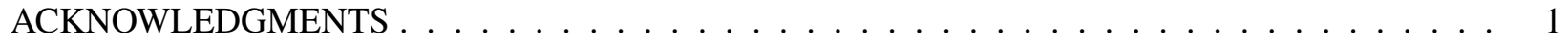

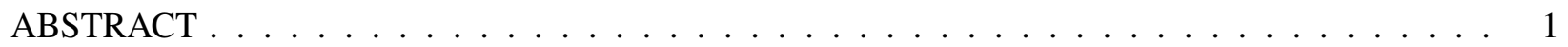

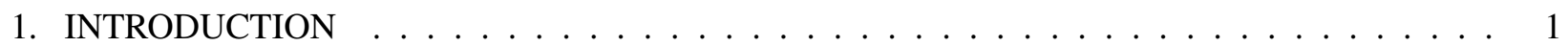

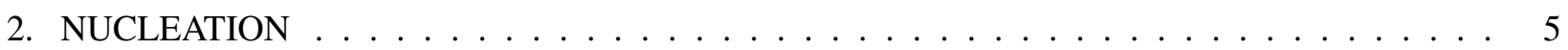

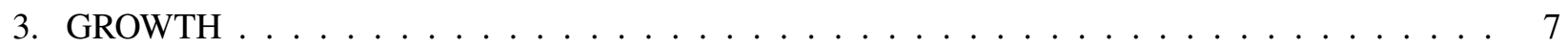

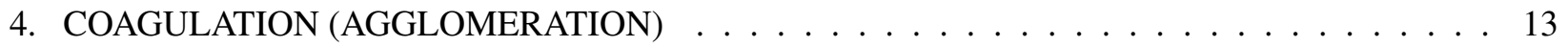

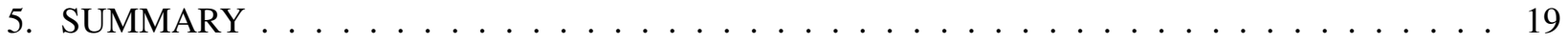

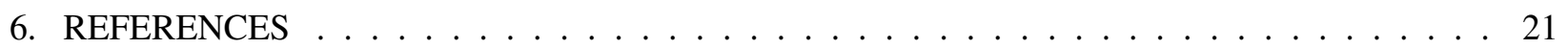





\section{LIST OF FIGURES}

1 Schematic representation of the sectional discretization of the particle size domain and the action of the nucleation, growth and agglomeration processes. . . . . . . . . . 3

2 Vapor pressure for a substance in a solution as a function of its molar fraction $(x) \ldots \ldots$

3 Description of the collision sphere of radius $R$ for the interaction of a particle with radius $r_{1}$ with a particle with radius $r_{2} \ldots \ldots \ldots \ldots \ldots \ldots$ 



\section{LIST OF SYMBOLS}

\begin{tabular}{ll}
$B_{i}$ & Barrier $i$ \\
$c_{p g}$ & Specific heat at constant pressure of gas \\
$c_{p}$ & Specific heat at constant pressure of vapor \\
$D_{v g}$ & Vapor diffusivity in gas \\
$F_{i}$ & Particle flux from section $i$ \\
$g$ & Gravitational acceleration \\
$g_{c}$ & Gibbs free energy per molecule in the condensed phase \\
$g_{g}$ & Gibbs free energy per molecule in the gas phase \\
$G$ & Gibbs free energy \\
$\dot{H}$ & Heat flux \\
$\dot{H}_{i n t}$ & Heat flux to the interior of the particle \\
$k_{B}$ & Boltzmann's constant \\
$K n$ & Knudsen number \\
$L$ & Latent heat \\
$\bar{m}$ & Mass of a molecule \\
$\dot{M}$ & Mass flux \\
$n$ & Number of molecules \\
$n_{i}$ & Number of particles in section $i$ \\
$N u_{H}$ & Nusselt number for heat transport \\
$N u_{M}$ & Nusselt number for mass transport \\
$P_{v}$ & Vapor pressure \\
$P_{0}$ & Saturation vapor pressure \\
$P_{s}$ & Saturation vapor pressure at particle surface \\
$P_{g}$ & Total gas pressure \\
$P\left(v_{r}\right)$ & Probability distribution of relative velocity between particles \\
$r$ & Particle radius \\
$R_{v}$ & Vapor individual gas constant \\
$R_{g}$ & Gas individual gas constant \\
$r_{i}$ & Characteristic radius of section $i$ \\
$S_{i}$ & Section $i$ \\
$S t k$ & Stokes number \\
$t$ & Time \\
$T_{g}$ & Gas temperature \\
$T_{s}$ & Particle surface temperature \\
$u_{i}$ & Characteristic volume of section $i$ \\
$V\left(x \mid x^{\prime}\right)$ & Collision kernel between sizes $x$ and $x^{\prime}$ \\
$V_{T}\left(x \mid x^{\prime}\right)$ & Collision kernel between sizes $x$ and $x^{\prime}$ due to turbulence \\
$V_{B}\left(x \mid x^{\prime}\right)$ & Collision kernel between sizes $x$ and $x^{\prime}$ due to Brownian motion \\
$V_{G}\left(x \mid x^{\prime}\right)$ & Collision kernel between sizes $x$ and $x^{\prime}$ due to gravity \\
$V_{D}\left(x \mid x^{\prime}\right)$ & Collision kernel between sizes $x$ and $x^{\prime}$ due to turbulence \\
& gravitational settling \\
& Aolar fration coefficient for evaporation \\
\hline &
\end{tabular}




$\begin{array}{ll}\alpha_{c} & \text { Accommodation coefficient for condensation } \\ \Delta r_{i} & \text { Radial width of section } i \\ \Delta m_{i}^{k} & \text { Mass change of species } k \text { in section } i \\ \Delta M_{v}^{k} & \text { Mass change of species } k \text { in the gas } \\ \epsilon_{g} & \text { Turbulent kinetic energy dissipation rate per unit mass } \\ \kappa & \text { Thermal conductivity of gas } \\ \lambda & \text { Mean free path } \\ \mu & \text { Molar mass } \\ \mu_{g} & \text { Dynamic viscosity of the gas } \\ v_{g} & \text { Kinematic viscosity of the gas } \\ v_{r} & \text { Relative velocity between particles } \\ \rho_{c} & \text { Density of condensed phase } \\ \rho_{c}^{k} & \text { Density of condensed phase for species } k \\ \rho_{g} & \text { Density of the gas } \\ \sigma & \text { Surface tension } \\ \Sigma & \text { Vapor saturation }\end{array}$




\title{
ACKNOWLEDGMENTS
}

This work was funded by the Office of Nuclear Detonation Detection - Forensics Program within the U. S. Department of Energy's National Nuclear Security Administration as part of the Water-Surface-Burst Fallout Model project.

\begin{abstract}
Formulations of the principal physical processes active during the formation of nuclear debris are described, with an emphasis on first principles approaches and moderate computational costs. The discretization of these models for implementation into numerical codes is also discussed.
\end{abstract}

\section{INTRODUCTION}

The capability to predict the characteristics and trajectories of debris particles formed after a nuclear burst plays an important role in the planning of emergency response and forensic analysis activities (Fahey et al. [2010]). The limited number of conditions for which nuclear tests were carried out means that parameterizations based on available experimental data may not be applicable to all the scenarios of interest, such as water-surface bursts. The use of first principles models is an attractive approach that can lead to the development of more generic descriptions of the evolution of nuclear debris.

The high initial temperatures in the fireball result in the complete vaporization of the constituents of the weapon and fission products and depending on the scenario, also of part of the surrounding structures. As the temperature of the fireball decreases, first by radiation and later by mixing with cooler ambient air, the species in the gas phase condense into droplets and particles.* The order in which these phase transformations occur is dictated by the intrinsic volatility of the species, their vapor concentration, and the existing condensed phase. The combination of the radioactive decay process and chemical kinetics leads to a temporal dependence of the composition of the system, thus affecting the level of supersaturation and the ability of the constituents of a given mass chain to condense. The rate the phase transformation occurs is also determined by the available substrate, as the saturation vapor pressure at the surface of particles will depend on their size and composition. As a result, the characteristics of the condensation process at a given time will be a function of its previous history, and models need to follow the evolution of the system from well before a condensed phase emerges.

The change in conditions with time in the fireball leads to an evolving population of debris particles, with size-dependent compositions and distributions that can be multimodal. The characteristics of particle samples collected at later times will reflect the convolution of the heterogeneous population in the fireball with the atmospheric transport process driving the particles to the point of collection. For example, the ratios of nuclide masses in the samples (after correction for radioactive decay) will not be representative of those present in the fireball as a whole at a given time. This fractionation phenomenon is a major complicating factor in the forensic analysis of samples, and models play an important role in the understanding of its origin and the development of operational techniques to account for it.

In this report we present a description of some of the important physical processes that directly determine the formation of nuclear debris. We consider these models to be a minimum capability able to satisfy the principal requirement, which is to compute the functional dependence of nuclide masses with particle size.

${ }^{*}$ For the rest of this report we will use the term particle to refer both to liquid and solid particles. 
A first principles approach has been favored here, and it has been balanced with the need for moderate computational cost. The main assumptions that have allowed for this are the restriction to local uniform conditions and the use of macroscopic descriptions based on a thermodynamic formalism. Under the former, we take the conditions of the system during the time interval of the computation to be spatially uniform in the volume under consideration, being characterized by a single gas temperature and composition. These models can be applied locally in a fully three-dimensional description that allows for spatial variations or used under the assumption that mixing in the fireball is intense enough for the conditions in it to be approximately uniform. Under this approach we also assume that, at a given time, all particles of a given size have the same composition. The adoption of a thermodynamic description derives from the current inability of more atomistic approaches to handle the spatial and temporal scales involved in this problem within a reasonable computational time. Studies at the more fundamental level still play a role in the understanding of the physical processes and the determination of material properties that are difficult to measure experimentally, and efforts are ongoing to incorporate this information into the macroscopic models through parameterizations.

We will focus here on three of the main processes involved in debris formation: nucleation, growth, and coagulation. By (homogeneous) nucleation we refer to the formation of a condensed phase from the vapor mix in the gas. Since this involves the appearance of an interface, there is an associated energy barrier that needs to be surmounted and usually requires substantial levels of supersaturation. In the case when a condensed phase already exists, further condensation on it (growth) is usually the most energy-favored path. Additionally, when the number of particles per unit volume is considerable, given the polydispersive character of the particle population and the high degree of turbulence in the flow, collisions and coalescence processes can have a profound effect on the overall population.

The temporal variations in the conditions of the systems also produce an approximate continuum of particle sizes. For computational purposes functional representations of the number and mass distributions need to be used and several approaches have been developed as part of the studies of other particle-forming systems (Zhang et al. [2020]). They can be classified as those that assume the general functional form of the size distributions and those that create a partitioning of the particle-size domain and only impose a functional dependence within each of those sections (bins). Here we follow the latter approach, usually described as sectional (Gelbard et al. [1980]), as its provides higher flexibility in the description of the particle population, which is essential in the case of nuclear debris because it is known that the size distributions are usually complex and can present multiple modes (Nathans et al. [1969]). Both approaches introduce errors derived from the discretizations that affect the calculated distributions with spurious dispersion or diffusion (Tsang and Rao [1988]), but we will consider these limitations to be acceptable within the degree of accuracy we are searching here. We adopt a sectional description with a functional dependence in each bin that corresponds to a delta function around a characteristic size, which means that in each partition all particles have the same dimension. This type of approach is sometimes called a nodal description (Prakash et al. [2003]).

As shown in Figure 1, we employ an arbitrary partitioning of the particle-size domain ${ }^{\dagger}$ by means of barriers $\left(B_{i}\right)$ that demarcate sections, and for each of them we adopt as the representative size their midpoint $\left(S_{i}\right)$, that is $S_{i}=B_{i}+\Delta S_{i} / 2$, with the width of the section given by $\Delta S_{i}=B_{i+1}-B_{i}$. In this formulation, the evolution of the distribution of material masses in each bin (and derived from it that of the particle number)

\footnotetext{
'By size here we mean a certain function of the volume of the particles.
} 


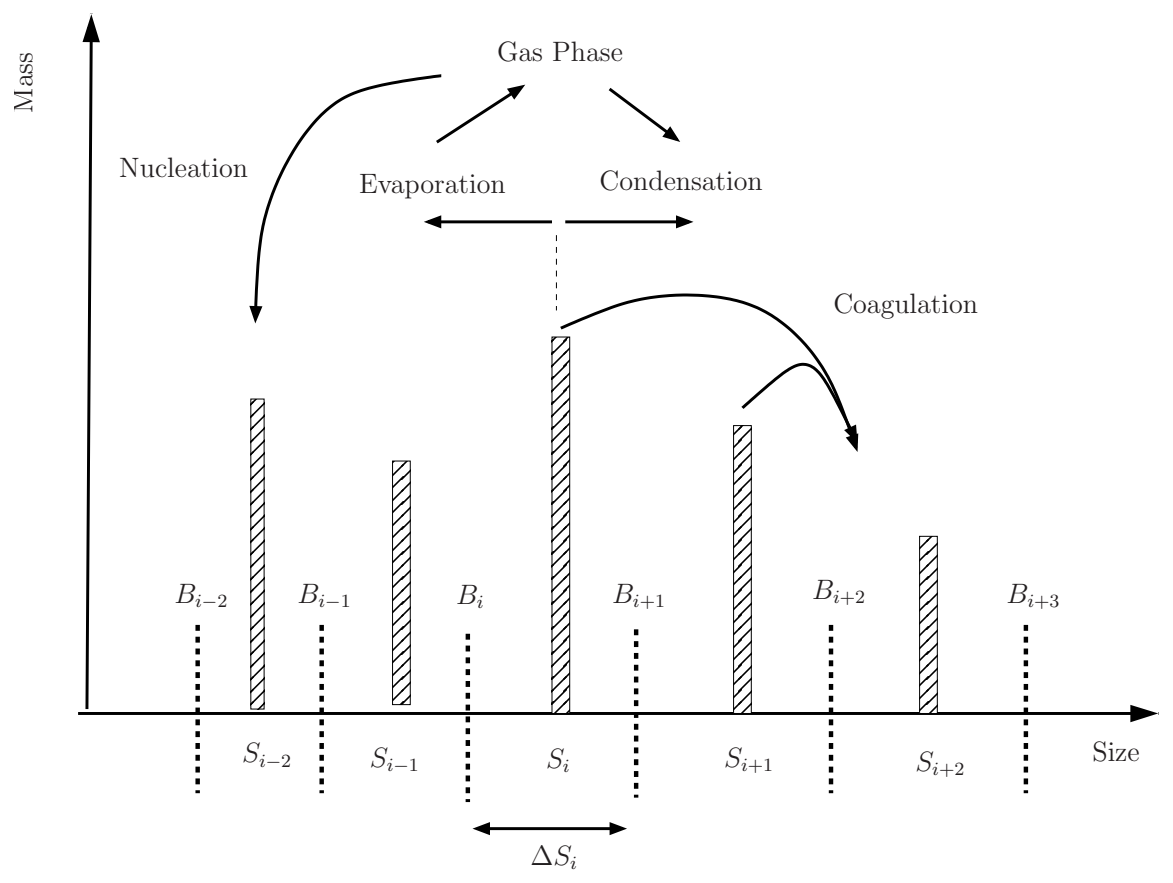

Figure 1. Schematic representation of the sectional discretization of the particle size domain and the action of the nucleation, growth and agglomeration processes.

satisfies an equation known as the general dynamic equation (Gelbard and Seinfeld [1979]),

$$
\frac{d m_{i}}{d t}=\left.\frac{d m_{i}}{d t}\right|_{\text {nucleation }}+\left.\frac{d m_{i}}{d t}\right|_{\text {growth }}+\left.\frac{d m_{i}}{d t}\right|_{\text {agglomeration }},
$$

where $t$ refers to time and $m_{i}$ is the mass (per unit volume) of a component in particles with size corresponding $S_{i}$.

The solution of this equation implies calculating the mass fluxes between sections and the gas phase, under the constraint of total mass conservation. The computed evolution of the debris population will necessarily lead to the formation of particles with sizes different from those represented in the bin distribution and this makes it necessary to adopt a remapping algorithm, which distributes those masses to existing sections.

One important characteristic of nuclear debris is its complex composition due to the multitude of fission products formed during the burst. Attempts have been made in the past to describe multicomponent systems by means of multidimensional size-distributions (Gelbard and Seinfeld [1980]), but they require working with a number of degrees of freedom on the order of the number of species times the number of size sections. For the number of nuclides and associated species that need to be considered in the modeling of nuclear debris these formulations become impractical. From the analysis of fallout particles from nuclear bursts there is evidence that particles of similar sizes have on average similar compositions and this is compatible with the assumption of well-mixed conditions in the fireball, except for bursts where there are substantial amounts of material entrained into the fireball (Adams et al. [1960], Magee [1953]). It is then justifiable to adopt the approximation that at a given time, particles within a given size bin have the same composition, an assumption that also forms the basis of widely used analytical approximations to nuclear debris characteristics (Freiling [1963]). Some refinements of this idea, with higher accuracy but 
more limited applicability, have been proposed for the description of similar multicomponent systems (Gelbard et al. [1998]), but we do not deem them suitable for the level of complexity and degree of generality we are pursuing here. In cases when the particle population continues evolving after thermal equilibrium with the atmosphere has been reached, for example when water condensation is important during the rise of the nuclear cloud, this assumption will not necessarily be valid. In that case, particles will follow different trajectories in the cloud and will experience different levels of humidity, resulting in different growth rates and compositions. Nevertheless, the approach described here will still be useful if applied locally for a short time interval and in combination with a Lagrangian description of the particles, where the changes in particle population due to condensation are mapped back to individual particles.

Below we describe, for each term in the general dynamic equation, the physical basis of the associated processes and the approximations required for a discrete representation. The mass fractions of the components in the fireball will in general be very dissimilar, with the concentrations of fission products several orders of magnitude smaller than those of the un-burnt fuel. Since some of those trace constituents may be of primary interest in the application of the models, care must be taken to select numerical implementations that are mass conserving. Given the discrete nature of the computational models, this requirement often results in the introduction of higher uncertainty in other variables, for example in the number of particles.

Besides the three main processes described here, other physical mechanisms will need to be taken into account for a complete description of the debris formation process. We only mention their requirements briefly since their implementation is highly dependent on the way these local models are linked to a global description.

In addition to nucleation, condensation, and agglomeration, it is necessary to have a good representation of the chemistry of the fireball, especially in the gas phase. At early time, chemical kinetics can be expected to be fast because of the high temperatures, but there is still substantial uncertainty about the reaction rates and species involved (Koroglu et al. [2018], Finko et al. [2017]). In the past, oxides were assumed to be the dominant species (Miller [1960]), but recently researchers have suggested the possibility of reducing conditions (Giuli et al. [2010], Cassata et al. [2014]). As a consequence, models need to include a description of the chemical kinetics and should be updated as research on the reaction pathways and rates progresses. Since the resolution of isotopic ratios will be an important aspect in many application of these models, care needs to be taken to preserve those ratios during the numerical solution of the chemical processes.

Another factor affecting the composition of the system stems from the radioactive decay of many of its constituents. A decay solver has to be included as part of the modeling and it needs to able to keep track of the mass fractions of a given nuclide that are associated with different species.

Although the approaches described here assume spatially uniform conditions, it is possible that mass exchanges with the exterior of the local volume may occur during the computation time. Examples are the partial precipitation of the condensed phase due to the action of gravity or the entrainment into the fireball of material from external structures, such as during the so called sweep-up mechanism in bursts close to the ground. The way these effects are implemented will depend on the specific characteristics of the global model used, but in terms of its relation to local models, in all cases the underlying assumption is that changes in the distribution of the condensed phase are spatially uniform in the volume of interest. 


\section{NUCLEATION}

The high temperatures reached in the fireball at early time make it reasonable to assume that all materials are in the gas form. The initial formation of a condensed phase or homogeneous nucleation will involve overcoming the energy barrier associated to the surface energy of the new nuclei, which usually requires vapor partial pressures corresponding with high levels of supersaturation.

Nucleated particles have typical radii of the order of nanometers and their properties are expected to be different from those measured macroscopically. Nevertheless, a description of the nucleation process at the atomistic level would be prohibitively expensive. At present, a viable alternative is to adopt the classical theory of nucleation as introduced by Volmer and Weber (Volmer and Weber [1926], Seinfeld and Pandis [1997]), which follows a statistical approach to describe the formation of metastable clusters by thermal fluctuations. The description uses macroscopic quantities to describe the properties of the condensed phase, for example representing the interface energy by means of the surface tension of the substance. It also treats the growth of embryos as occurring one molecule at a time, while ignoring the depletion of the gas phase.

If the nuclei are deemed to be spherical with radius $r$ and containing $n$ molecules of mass $\bar{m}$, we can write:

$$
n=\frac{4 \pi}{3} \frac{r^{3} \rho_{c}}{\bar{m}}
$$

where $\rho_{c}$ is the macroscopic density of the condensed phase.

The Gibbs free energy $(G)$ of the forming nuclei can be decomposed into bulk and surface components and it is possible to write the change of energy associated with the formation of a nucleus as

$$
\begin{array}{r}
\Delta G=\left(g_{c}-g_{g}\right) \frac{4 \pi r^{3} \rho_{c}}{3 \bar{m}}+4 \pi r^{2} \sigma= \\
\left(g_{c}-g_{g}\right) n+\sigma(4 \pi)^{1 / 3}\left(\frac{3 \bar{m} n}{\rho_{c}}\right)^{2 / 3},
\end{array}
$$

where $g_{c}$ and $g_{g}$ are the free energies per molecule in the condensed and gas phases, respectively, and $\sigma$ is the surface tension.

For conditions of supersaturation, $g_{c}<g_{g}$ and the first term in (3) will be negative, whereas the second term is always positive. This results in a critical value for the number of molecules in the nucleus, $n^{*}$, corresponding to a maximum of $\Delta G$. The system must progress so as to lower $\Delta G$ and clusters with $n>n^{*}$ are stable and will continue to grow, while those with $n<n^{*}$ will evaporate.

If the phase transformation occurs at constant temperature, the gas phase is considered ideal and the density of the vapor negligible compared to that of the condensed phase, it is possible to write (Seinfeld and Pandis [1997])

$$
g_{c}-g_{g}=-k_{B} T_{g} \ln \Sigma,
$$

where $k_{B}$ is Boltzmann's constant, $T_{g}$ is the temperature of the gas, and $\Sigma$ is the supersaturation of the vapor, i.e., the ratio of its partial pressure $\left(P_{v}\right)$ to its saturation value over an infinite plane $\left(P_{0}\right)$.

By differentiating (3) with respect to $n$ or $r$ and equating to zero we can find the critical radius and number of molecules for the embryos,

$$
r^{*}=\frac{2 \sigma}{k_{B} T_{g} \ln \Sigma} \frac{\bar{m}}{\rho_{c}}, \quad n^{*}=\sigma^{3} \frac{32}{3} \pi\left(\frac{\bar{m}}{\rho_{c}}\right)^{2} \frac{1}{\left(k_{B} T_{g} \ln \Sigma\right)^{3}} .
$$


For a system with $N_{0}$ molecules, under the assumption that a population of clusters exists that is in equilibrium with the saturated vapor, it is possible to show that the cluster sizes will follow a Boltzmann distribution (Feder et al. [1966])

$$
N(n)=N_{0} \exp \left(-\frac{\Delta G}{k_{B} T_{g}}\right)
$$

and the number of embryos of critical size satisfies

$$
N\left(n^{*}\right)=N_{0} \exp \left[\frac{16 \pi}{3}\left(\frac{\bar{m}}{\rho_{c} \ln \Sigma}\right)^{2}\left(\frac{\sigma}{k_{B} T_{g}}\right)^{3}\right] .
$$

The nuclei of critical size are in metastable equilibrium and by the addition of an extra molecule they become stable. The rate at which these collisions occur can be determined from the kinetic theory of gases, and under the assumption that all collisions lead to coagulation, it is found that the rate at which stable nuclei are formed per unit time and volume is

$$
J=\frac{\mu}{\Sigma \rho_{c}}\left(\frac{P_{v}}{k_{B} T}\right)^{2}\left(\frac{2 \sigma}{\pi \bar{m}}\right)^{1 / 2} \exp \left[\frac{16 \pi}{3}\left(\frac{\bar{m}}{\rho_{c} \ln \Sigma}\right)^{2}\left(\frac{\sigma}{k_{B} T_{g}}\right)^{3}\right]
$$

where $R_{v}$ is the individual gas constant and $\mu$ the molar mass of the condensing substance. Different forms of the factor multiplying $N\left(n^{*}\right)$ in (8) have been derived, for example by relaxing condition (6) (Girshick and Chiu [1990]), but overall the nucleation rates predicted by the classical theory can differ by several orders of magnitude with experimental results. Given the uncertainties in the existing formulations, we will refrain from considering further theoretical refinements, and we will also neglect the possibility of heteromolecular nucleation, where more than one substance nucleate simultaneously.

In the discrete approximation to the particle population, in general the size of the nucleated particles will not correspond to any of the characteristic sizes of the partitions. It is then necessary to associate the particles to one of the existing bins, and it is common practice to add them to the closest partition with a smaller characteristic radius. The mass added to the condensed phase is that computed with (8) and the inexact radius used introduces an error in the size and number of the particles created. The mass nucleated is then deducted from the vapor mass to maintain mass conservation. 


\section{GROWTH}

The rate of mass transfer of a substance from the gas phase to a single particle depends on the degree of saturation of the corresponding vapor and the characteristics of the substrate. The latter affect the saturation vapor pressure at the surface of the particle, mainly because of its curvature, the ability to react chemically, or the ability to form a solution.

Although phase transformations require net fluxes of mass and heat and the system is not in equilibrium, depending on the time scales involved it is sometimes possible to adopt a description that assumes that the profiles of concentration and temperature have reached a steady state. This has been shown to typically occur in a time scale much smaller than the characteristic condensation times (Frisch and Collins [1952, 1953]), which in our case are also usually much shorter than typical cooling rates.

The condensation (evaporation) of a substance at the surface of the particle results in the release (absorption) of latent heat, which will alter the temperature of the interface and hence the saturation vapor pressure and the flux of mass from the gas phase. The steady state is attained when fluxes satisfy

$$
\dot{H}=\dot{H}_{\text {int }}-L \dot{M},
$$

where $\dot{H}$ and $\dot{M}$ are the heat and mass fluxes between the gas and the particle, $\dot{H}_{\text {int }}$ is the heat flux toward the interior of the particle and $L$ the latent heat of condensation. Except during the period of thermalization of particles entrained into the fireball, in general $\dot{H}_{\text {int }} \ll \dot{H}$ (Gyarmathy [1982]), and the heat and mass fluxes can be considered to be proportional:

$$
\dot{H}=-L \dot{M} \text {. }
$$

Given the particle characteristics, the vapor concentration and temperature in the gas phase, equation (10) can be considered an implicit equation for the temperature at the interface $\left(T_{s}\right)$.

The characteristic length of the system is the mean free path in the gas phase and different regimes can be identified in terms of the size of the particles in relation to it (Fuchs [1959], Wright [1960]). This is usually quantified in terms of the Knudsen number $(K n)$ :

$$
K n=\lambda / r,
$$

where $\lambda$ is the mean free path and $r$ the radius of the particle.

Analytical expressions can be obtained for the fluxes in the limits of large and small $K n$ (Seinfeld and Pandis [1997]). For $K n \ll 1$, the gas can be seen as a continuum and the mass transport as a diffusion process; consequently, the fluxes can be written as

$$
\begin{aligned}
\dot{M} & =-4 \pi r D_{v g} \frac{\left(P_{v}-P_{s}\right)}{R_{v} T_{g}}, \\
\dot{H} & =4 \pi r \kappa\left(T_{s}-T_{g}\right),
\end{aligned}
$$

where $D_{v g}$ is the diffusivity of the condensing vapor in the carrier gas, $\kappa$ the thermal conductivity and $P_{v}$ the partial vapor pressure in the gas phase

\footnotetext{
${ }^{\ddagger}$ When the condensing vapors are very dilute in the gas phase, the mass advection by the flow can be neglected and the fluxes are determined by diffusion (Gyarmathy [1982]).
} 
On the other hand, for small particles (i.e., $K n \gg 1$ ) condensation can be described in terms of the interaction of individual gas molecules with the substrate and in this case the fluxes take the form

$$
\begin{aligned}
& \dot{M}=4 \pi r^{2}\left(\frac{\alpha_{e} P_{s}}{\sqrt{2 \pi R_{v} T_{s}}}-\frac{\alpha_{c} P_{v}}{\sqrt{2 \pi R_{v} T_{g}}}\right), \\
& \dot{H}=4 \pi r^{2}\left[\frac{P_{v}\left(c_{p}-\frac{1}{2} R_{v}\right)}{\sqrt{2 \pi R_{v} T_{g}}}+\frac{P_{g}\left(c_{p g}-\frac{1}{2} R_{g}\right)}{\sqrt{2 \pi R_{g} T_{g}}}\right]\left(T_{s}-T_{g}\right),
\end{aligned}
$$

where $P_{g}, c_{p g}$ and $R_{g}$ are the pressure, specific heat, and gas constant of the gas, respectively; $c_{p}$ is the specific heat of the condensing substance; and $\alpha_{c}$ and $\alpha_{e}$ the accommodation coefficients for condensation and evaporation, here taken to be unity.

As multiple physical processes are involved in the evolution of the debris population, which results in a wide range of particle sizes, it is necessary to have a functional description for the fluxes at intermediate values of $\mathrm{Kn}$. There are different approaches to achieving this, and here we follow the formulation by Gyarmathy (Gyarmathy [1982], Peeters et al. [2001]), who writes the fluxes in terms of generalized Nusselt numbers

$$
\begin{aligned}
\dot{M} & =4 \pi r^{2} N u_{M}(K n) \frac{\left(P_{s}-P_{v}\right)}{P_{g}} \frac{D_{v g}^{*}}{2 r}, \\
\dot{H} & =4 \pi r^{2} N u_{H}(K n)\left(T_{s}-T_{g}\right) \frac{\kappa}{2 r},
\end{aligned}
$$

where $D_{v g}^{*}=D_{v g} P_{g} / R_{v} T_{m}$ and $N u_{H}$ and $N u_{M}$ are the Nusselt numbers associated with heat and mass transport and whose functional dependence on $K n$ is chosen so that $\dot{M}$ and $\dot{H}$ tend to the right limits when $K n \rightarrow 0$ and $K n \rightarrow \infty$. In the continuum limit this corresponds to

$$
N u_{M}^{c}=2, \quad N u_{H}^{c}=2,
$$

whereas in the molecular kinetics limit

$$
\begin{aligned}
& N u_{M}^{m}=\frac{2 r R_{v} T_{m}}{D_{v g}\left(P_{s}-P_{v}\right)}\left[\frac{\alpha_{e} P_{s}}{\sqrt{2 \pi R_{v} T_{s}}}-\frac{\alpha_{c} P_{v}}{\sqrt{2 \pi R_{v} T_{g}}}\right], \\
& N u_{H}^{m}=\frac{2 r}{\kappa}\left[\frac{P_{v}\left(c_{p}-\frac{1}{2} R_{v}\right)}{\sqrt{2 \pi R_{v} T_{g}}}+\frac{P_{g}\left(c_{p g}-\frac{1}{2} R_{g}\right)}{\sqrt{2 \pi R_{g} T_{v}}}\right],
\end{aligned}
$$

with $T_{m}=\left(2 T_{s}+T_{g}\right) / 3$.

For intermediate values of $K n$ we adopt the mapping (Gyarmathy [1982])

$$
N u_{M}=\frac{N u_{M}^{c} N u_{M}^{m}}{N u_{M}^{c}+N u_{M}^{m}}, \quad N u_{H}=\frac{N u_{H}^{c} N u_{H}^{m}}{N u_{H}^{c}+N u_{H}^{m}} .
$$

The vapor pressure on the surface of the particle depends on its composition. In particular, if the condensing substance is able to form a solution with all or part of the condensed phase, this can result in a reduced $P_{s}$ and a lower degree of supersaturation for condensation to occur. Simple analytical descriptions of the properties of binary solutions are possible if the involved substances can be clearly classified as the 


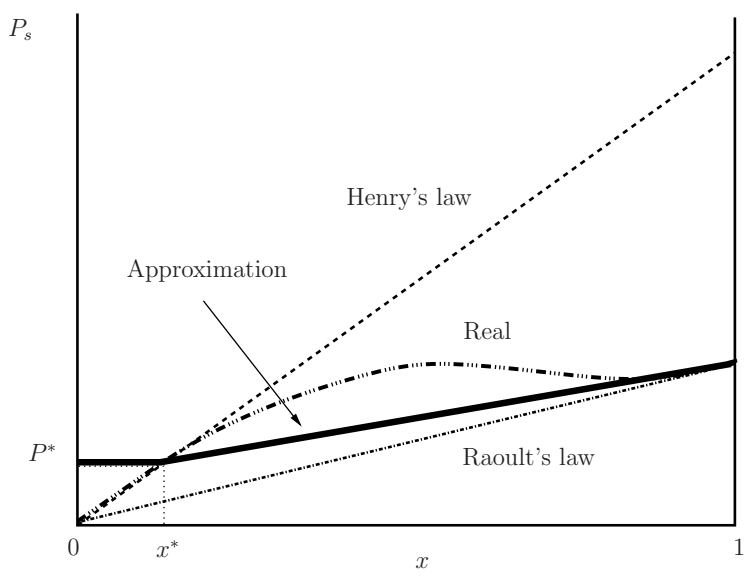

Figure 2. Vapor pressure for a substance in a solution as a function of its molar fraction $(x)$.

solvent and solute, under the assumption that one of them is in a much smaller concentration than the other (Atkins [1986]).

When the condensing substance can be considered to be the dilute solute, it is common practice to use Henry's law, where its vapor pressure in the solution is given by

$$
P_{s}=x H,
$$

with $x$ the molar fraction of the solute and $H$ its Henry's law constant, which is in general determined experimentally.

On the other hand, when the condensing substance is the most abundant in the solution, e.g., vaporized soil in a surface burst, its vapor pressure is described by Raoult's law,

$$
P_{s}=x P_{0},
$$

where $x$ is now the molar fraction of the solvent and $P_{0}$ its vapor pressure over the pure substance.

Solutions for which Raoult's law is satisfied for all concentrations are called ideal. In a real system, the vapor pressure will show a dependence on the mole fraction that asymptotes to the two linear approximations for the corresponding limits in $x$ (Figure 2).

During the formation of nuclear debris, materials condense at different times depending on the volatility of the species and their concentration. Depending on the scenario, it is likely that the most abundant components, such as those associated to the casing of the weapon, will condense on an existing particle population of more refractory species and will become the "matrix" of the particle. The models are then required to be able to continuously handle the possibility that the condensing species forms a solution with the substrate with a molar fraction that over time covers the range from $x \approx 0$ to $x \approx 1$. The model described here is a generalized description that also covers the case of a solid substrate and allows for conditions where, at low concentrations, the condensing substance forms a saturated solution with part of the substrate corresponding to a mole fraction $x^{*}$ and pressure $P^{*}$ (e.g., the case of water condensing on $\mathrm{NaCl}$ crystals), and that for larger concentrations the relation is linear with $x$ (Figure 2). In this way, the adjustment of the parameters $x^{*}$ and $P^{*}$ makes it possible to prescribe vapor curves that follow Henry's or Raoult's laws, or that provide a better approximation to the real vapor curve for a wide range of molar fractions. 
Another factor affecting the vapor pressure is the curvature of the surface of the particle, which is classically described by Kelvin's law (Kelvin [1870]),

$$
P_{s}=P_{0} \exp \left(\frac{2 \sigma}{r R_{v} \rho_{c} T}\right)
$$

where $P_{0}$ is the saturation vapor pressure over an infinite flat surface.

Replacing (16) and (17) into (10) yields

$$
N u_{H} \kappa\left(T_{g}-T_{s}\right)=-N u_{M} L D_{v g}^{*} \frac{\left(P_{v}-P_{s}\right)}{P_{g}},
$$

which can be solved for $T_{s}$ and hence $P_{s}$. From the mass flux to the particles it is possible to obtain the change in their radius with time. Using (16), (17) and (10) this can be written,

$$
\begin{aligned}
& \frac{d r}{d t}=\frac{N u_{H} \kappa}{2 r \rho_{c} L}\left(T_{s}-T_{g}\right), \\
& \frac{d r}{d t}=-\frac{N u_{M} D_{v g}^{*}}{2 r \rho_{c}} \frac{\left(P_{s}-P_{v}\right)}{P_{g}} .
\end{aligned}
$$

These quantities are equivalent to a "velocity" along the particle-size axis. If we define a partitioning in terms of particle radius, with bin $i$ having characteristic radius $r_{i}$ and width $\Delta r_{i}$, the flux of particles across a barrier $B_{i+1}$ can be written as

$$
F_{i}=\frac{d r_{i}}{d t} \frac{n_{i}}{\Delta r_{i}}
$$

where $n_{i}$ is the number of particles contained in the $i$ section.

In the case of condensation, $F_{i}>0$ and particles will move toward a bin of larger size. During the time interval $\Delta t,{ }^{\S}$ the mass of component $k$ added to partition $r_{i+1}$ and originating from the vapor phase satisfies, apart from a factor,

$$
\Delta m_{i}^{k} \propto \frac{F_{i}\left(u_{i+1}-u_{i}\right)}{\rho_{c}^{k}} \Delta t
$$

where $u_{i}$ is the characteristic volume for partition $i$ and $\rho_{c}^{k}$ is the density of the condensed phase for substance $k$.

In the case of a negative flux or evaporation, the equivalent expression is

$$
\Delta m_{i}^{k} \propto \frac{F_{i}\left(u_{i}-u_{i-1}\right)}{\rho_{c}^{k}} \Delta t
$$

and now $\Delta m_{i}^{k}$ is the mass transferred from bin $i$ to the gas.

At each time step, conservation of mass for species $k$ then takes the form

$$
\sum_{i} \Delta m_{i}^{k}=\Delta M_{v}^{k}
$$

\footnotetext{
${ }^{\S}$ We assume that $\Delta t$ is small enough for particles not to move beyond adjacent bins during one time step.
} 
where $\Delta M_{v}^{k}$ is the total change in mass for the vapor during $\Delta t$.

As the mass and heat flux equations are nonlinear and a function of all the species in the system, their numerical integration can result in instabilities. In this implementation, and for a given time step, we find the solution for each species sequentially. This can be taken to be a linearization of the full equations, and the solution will be a first order approximation.

The fluxes between bins described above need to take into account also the species that are not condensing but are already present in the particles and moving with them. This means that when condensation occurs, bin $i$ experiences an additional change in mass given by

$$
\Delta m_{i}=\sum_{l} \frac{F_{i}}{n_{i}} m_{i}^{l} \Delta t,
$$

where the sum is taken over all species. In the case of evaporation, the equivalent relation is

$$
\Delta m_{i}=\sum_{l} \frac{F_{i}}{n_{i}} m_{i}^{l} \Delta t-\Delta m_{i}^{k},
$$

where we discounted the mass lost to the gas phase. 



\section{COAGULATION (AGGLOMERATION)}

The collision and coalescence of particles can have an important effect in the evolution of the overall population. Here we will only consider the coalescence of particles in pairs. The rate at which two particles merge into one, per unit time and volume, is usually decomposed into three factors (Saffman and Turner [1956], Wang et al. [1998]). One of these, the collision kernel, describes the rate at which the particles are driven to the same local region by the inhomogeneities in the velocity field. Here we will neglect the effect of the particles on the macroscopic flow, that is the coupling is taken to be only one way. Numerical studies have shown this assumption to be valid for particle volume fractions that are approximately below $10^{-4}$ (Elghobashi and Truesdell [1993], Ferrante and Elghobashi [2003]). Nevertheless, this approximation does not apply when the particles are in close proximity and modify the local flow velocity. It is customary to take this effect into account by introducing a second factor, the collision efficiency. Once two particles collide, factors such as particle size, composition, and surface forces (e.g., van der Waals and electrostatic), will determine if they coalesce into a single particle. This is known as the coalescence efficiency.

In our model we take the coalescence efficiency to be unity if both particles are in a liquid state or at least one of them has a radius below a critical value (we do not differentiate between coagulation and aggregation), otherwise we set its value to zero. Parameterizations of the collision efficiency for certain regimes have been obtained (Davies and Sartor [1967], Wang et al. [2005]), but this is still an area of research and in general we give this factor also a unit value. Below we focus on the formulation of the collision kernel.

In a continuous description, the number of particles of size $x$ per unit volume as a function of time varies as a result of the coagulation process according to

$$
\begin{array}{r}
\frac{\partial n(x, t)}{\partial t}=\int_{x_{0}}^{x / 2} n\left(x-x^{\prime}, t\right) V\left(x-x^{\prime} \mid x^{\prime}\right) n\left(x^{\prime}, t\right) d x^{\prime} \\
-\int_{x_{0}}^{\infty} n(x, t) V\left(x \mid x^{\prime}\right) n\left(x^{\prime}, t\right) d x^{\prime},
\end{array}
$$

where $x_{0}$ is the minimum size of the distribution (Smoluchowski [1917]). The first term on the right side describes the contribution to particles of size $x$ from the coalescence of pairs of particles smaller than $x / 2$, and the second term represents the loss of particles of size $x$ due to their interaction with other particles. The kernel $V\left(x \mid x^{\prime}\right)$ gives the probability of collision between a particle of size $x$ with a particle of size $x^{\prime}$, and it represents the contributions of the different physical processes responsible for the relative velocity between two particles, which drives them to the same region in space. In general terms, for two spherical particles with radii $r_{1}$ and $r_{2}$, it can be written as

$$
V\left(r_{1} \mid r_{2}\right)=2 \pi R^{2} \int\left|v_{r}\right| P\left(v_{r}\right) d v_{r}
$$

where $R=r_{1}+r_{2}, v_{r}$ is the relative velocity between the particles, and $P\left(v_{r}\right)$ the associated probability distribution (Saffman and Turner [1956]). As shown schematically in (Figure 3), in a frame of reference fixed on the particle with radius $r_{1}$, the kernel represents the integral of the flux of particles with radius $r_{2}$ across the sphere with radius $R$.

In the modeling of the evolution of nuclear debris it is necessary to take into account, at a minimum, the relative particle velocities originating from velocity gradients in the flow, gravitational settling and 


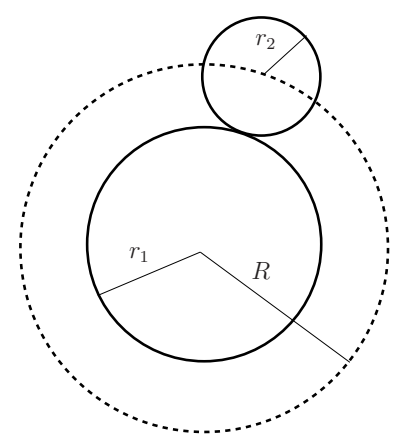

Figure 3. Description of the collision sphere of radius $R$ for the interaction of a particle with radius $r_{1}$ with a particle with radius $r_{2}$.

Brownian motion. There is no analytical expression for $P\left(v_{r}\right)$ that accounts for all these effects, and it is a common practice to formulate individual contributions to the kernel and add them together. Nevertheless, for this approach to be accurate, one of the terms in the expression for the kernel needs to be much larger in magnitude than the rest (Butuirat and Kielkiewickz [1996]).

For the relative motion between particles caused by Brownian motion (Chandrasekhar [1943]) different expressions can be derived as a function of the particle size with respect to the mean free path in the gas. In the continuum regime, the kernel can be written (Seinfeld and Pandis [1997])

$$
V_{B}\left(r_{1} \mid r_{2}\right)=4 \pi\left(r_{1}+r_{2}\right)\left(D_{1}+D_{2}\right)
$$

where $D$ is a diffusivity for particle $i$ defined by

$$
D_{i}=\frac{k_{B} T_{g}}{6 \pi r_{i} \mu_{g}}
$$

where $\mu_{g}$ is the dynamic viscosity of the gas.

In the molecular kinetics limit, the corresponding expression is

$$
V_{B}\left(r_{1} \mid r_{2}\right)=\pi\left(r_{1}+r_{2}\right)^{2} \sqrt{v_{1}^{2}+v_{2}^{2}},
$$

with

$$
v_{i}=\sqrt{\frac{8 k_{B} T_{g}}{\pi M_{i}}}
$$

where $M_{i}$ is the mass of particle $i$.

As it was done earlier for the condensation fluxes, expressions for the collision kernel have been proposed that are a function of the Knudsen number and valid for particles of all sizes (Fuchs [1959])

$$
V_{B}\left(r_{1} \mid r_{2}\right)=4 \pi\left(r_{1}+r_{2}\right)\left(D_{1}+D_{2}\right)\left[\frac{r_{1}+r_{2}}{r_{1}+r_{2}+\sqrt{\delta_{1}^{2}+\delta_{2}^{2}}}+\frac{4\left(D_{1}+D_{2}\right)}{\sqrt{v_{1}^{2}+v_{2}^{2}}\left(r_{1}+r_{2}\right)}\right]^{-1},
$$


with

$$
\delta_{i}=\frac{\left(2 r_{i}+\lambda_{i}\right)^{3}-\left(4 r_{i}^{2}+\lambda_{i}^{2}\right)^{3 / 2}}{6 r_{i} \lambda_{i}}-2 r_{i}, \lambda_{i}=\frac{8 D_{i}}{\pi v_{i}},
$$

and

$$
D_{i}=\frac{k_{B} T_{g}}{6 \pi \mu_{g} r_{i}}\left(\frac{5+4 K n_{i}+6 K n_{i}^{2}+18 K n_{i}^{3}}{5-K n_{i}+(8+\pi) K n_{i}^{2}}\right) .
$$

The difference in velocity between a particle of radius $r$ and density $\rho_{c}$ and the surrounding gas due to its inertia can be characterized in terms of the Stokes response time

$$
\tau_{p}=\frac{2 r^{2} \rho_{c}}{9 v_{g} \rho_{g}},
$$

where $v_{g}$ and $\rho_{g}$ are the kinematic viscosity and density of the gas.

Locally and for the particle size ranges relevant to nuclear debris calculations, variations in the velocity field due to turbulence can play an important role. At the dissipative scale the characteristic time $\left(\tau_{g}\right)$ and length scales $\left(\eta_{g}\right)$ can be defined as

$$
\tau_{g}=\sqrt{\frac{v_{g}}{\epsilon_{g}}}, \quad \eta_{g}=\left(\frac{v_{g}^{3}}{\epsilon_{g}}\right)^{1 / 4},
$$

where $\epsilon_{g}$ is the turbulent kinetic energy dissipation rate per unit mass. The importance of the particle inertia can be quantified using the Stokes number:

$$
S t k=\frac{\tau_{p}}{\tau_{g}} .
$$

For the interactions driven by turbulence, in the limit $S t k \rightarrow 0$ the particles move with the gas flow, but for Stk $\rightarrow \infty$ inertia is important and particle trajectories deviate substantially from the local streamlines. Particles with $S t k \approx 1$ tend to accumulate in regions of high strain and low vorticity (Maxey [1987]), leading to higher local concentrations and an enhancement of the collision kernel. Modifications to the coagulation models to account for this effect have been proposed (Wang et al. [2005]).

If the turbulence can be taken to be isotropic, for small Stokes numbers the collision kernel takes the form (Saffman and Turner [1956])

$$
V_{T}\left(r_{1} \mid r_{2}\right)=R^{3}\left(\frac{8 \pi \eta_{g}}{15 v_{g}}\right)^{1 / 2}
$$

The other factors contributing to the collision rate are the shear in the velocity field and gravitational effects. Under the local volume approximation we follow here, we will assume that the former is taken into account in the computation of the global particle trajectories, and it is not included explicitly in the expression of the collision kernel. In terms of gravitational effects, the kernel contribution can be written

$$
V_{G}\left(r_{1} \mid r_{2}\right)=\pi R^{2} g\left|\tau_{p, 1}-\tau_{p, 2}\right|
$$


where $\tau_{p, i}$ is the Stokes response time (43) for particle $i$.

Dodin and Elperin (Dodin and Elperin [2002]), assuming that the density of the particles is much larger than that of the gas and the only forces acting on the particles are drag and gravity, proposed a unified expression for the kernel that takes into account both turbulence and gravitational effects,

$$
V_{D}\left(r_{1} \mid r_{2}\right)=\sqrt{8} \pi\left(r_{1}+r_{2}\right)^{2} \beta f(b)
$$

with

$$
\begin{array}{r}
b=g\left|\tau_{p, 1}-\tau_{p, 2}\right| / \beta \sqrt{2}, \\
f(b)=0.5 \sqrt{\pi}(b+0.5 / b) \operatorname{erf}(b)+0.5 \exp \left(-b^{2}\right), \\
\beta=\gamma^{2}\left(r_{1}+r_{2}\right)^{2}+\lambda^{2}\left(\tau_{p, 1}-\tau_{p, 2}\right)^{2}, \\
\gamma^{2}=\frac{\epsilon_{g}}{15 v_{g}}, \quad \lambda^{2}=1.3 \frac{\epsilon_{g}^{3 / 2}}{v_{g}{ }^{1 / 2}} .
\end{array}
$$

In these expressions, the contribution to the kernel from turbulence effects becomes especially important for pairs of particles of the same size and density, where the contribution from gravitational effects cancels.

Here we approximate the total collision kernel by the sum of the two contributions,

$$
V\left(r_{1} \mid r_{2}\right)=V_{D}\left(r_{1} \mid r_{2}\right)+V_{B}\left(r_{1} \mid r_{2}\right)
$$

Depending on the regime, the functional dependence of $V_{D}$ on $R$ follows $R^{\alpha}$ with $2 \lesssim \alpha \lesssim 3$, whereas for $V_{B}$ the dependence on $R$ is of lower order, with $1 / 2 \lesssim \alpha \lesssim 1$. This shows that the magnitude of the kernels is very different for large and small particles, and (53) is an adequate description of the combined kernel. On the other hand, the contributions by $V_{D}$ and $V_{B}$ are of the same magnitude for particles with radii of the order of a micron (Seinfeld and Pandis [1997]), where also the total kernel tends to show a minimum. This is compatible with observations that particles originating in nucleation rarely grow beyond a few microns and that naturally occurring aerosol populations tend to present a minimum for those size ranges (Seinfeld and Pandis [1997]).

In a discrete representation of the particle size distribution using $N$ bins, it follows from (34) that the change in the number of particles in bin $i$ obeys

$$
\frac{d n_{i}}{d t}=\frac{1}{2} \sum_{\alpha=1}^{i-1} V\left(r_{i-\alpha} \mid r_{\alpha}\right) n_{i-\alpha} n_{\alpha}-n_{i} \sum_{\alpha=1}^{N} V\left(r_{i} \mid r_{\alpha}\right) n_{\alpha} .
$$

The finite-differences integration of (54) gives the particle number at time $t+1\left(n^{t+1}\right)$ as a functions of its value at time $t\left(n^{t}\right)$ by

$$
n_{i}^{t+1}=n_{i}^{t}+\frac{1}{2} \Delta t \sum_{\alpha=1}^{i-1} V\left(r_{i-\alpha} \mid r_{\alpha}\right) n_{i-\alpha}^{t+1} n_{\alpha}^{t+1}-\Delta t \sum_{\alpha=1}^{N} V\left(r_{i} \mid r_{\alpha}\right) n_{i}^{t+1} n_{\alpha}^{t+1}
$$


Numerical solutions of (55) need to be obtained iteratively because both sides of the equation depend on values at time $t+1$. To reduce computational costs, we adopted a semi-implicit approach by Jacobson and colleagues (Jacobson et al. [1994]), where $n_{\alpha}^{t+1}$ on the right side of the equation is replaced by $n_{\alpha}^{t}$ :

$$
n_{i}^{t+1}=n_{i}^{t}+\frac{1}{2} \Delta t \sum_{\alpha=1}^{i-1} V\left(r_{i-\alpha} \mid r_{\alpha}\right) n_{i-\alpha}^{t+1} n_{\alpha}^{t}-\Delta t \sum_{\alpha=1}^{N} V\left(r_{i} \mid r_{\alpha}\right) n_{i}^{t+1} n_{\alpha}^{t}
$$

Although the right side of (56) still depends on quantities at time $t+1$, the procedure is effectively explicit if the computation is carried out sequentially for increasing particle sizes;

$$
n_{i}^{t+1}=\frac{n_{i}^{t}+\frac{1}{2} \Delta t \sum_{\alpha=1}^{i-1} V\left(r_{i-\alpha} \mid r_{\alpha}\right) n_{i-\alpha}^{t+1} n_{\alpha}^{t}}{1+\Delta t \sum_{\alpha=1}^{N} V\left(r_{i} \mid r_{\alpha}\right) n_{\alpha}^{t}} .
$$

Because in our case particles of different sizes have different compositions and we want to impose mass conservation, instead of (57) we use an equivalent scheme that has been shown to conserve volume at the expense of some error in the particle number (Jacobson et al. [1994]). If we denote by $u_{k, i, t}$ the volume of component $k$ in size-bin $i$ at time $t$, then its value at time $t+1$ is obtained from

$$
u_{k, i, t+1}=\frac{u_{k, i, t}+\Delta t \sum_{\alpha=1}^{i}\left(\sum_{\beta=1}^{i-1} f_{\alpha, \beta, i} V\left(r_{\alpha} \mid r_{\beta}\right) u_{k, \alpha, t+1} n_{\beta, t}\right)}{1+\Delta t \sum_{\alpha=1}^{N}\left[\left(1-f_{i, \alpha, i}\right) V\left(r_{i} \mid r_{\alpha}\right) n_{\alpha, t}\right]},
$$

with

$$
U_{\alpha, \beta}=u_{\alpha}+u_{\beta},
$$

and

$$
f_{\alpha, \beta, i}= \begin{cases}\left(\frac{u_{i+1}-U_{\alpha, \beta}}{v_{i+1}-u_{i}}\right) \frac{u_{i}}{U_{\alpha, \beta}} & u_{i} \leq U_{\alpha, \beta}<u_{i+1}, \quad i<N, \\ 1-f_{\alpha, \beta, i-1} & u_{i-1}<U_{\alpha, \beta}<u_{i}, \quad i>1, \\ 1 & U_{\alpha, \beta} \geq u_{i}, \quad i=N, \\ 0 & \text { other cases, }\end{cases}
$$

where the factor $f_{\alpha, \beta, i}$ expresses the mapping of the particles formed by coagulation to the sizes available from the discretization.

In general, if the collection efficiency II is not zero, the coagulation process will lead to the creation of particles of increasing sizes. One way to deal with the evolving particle-size range within a sectional approach is to use moving partitions, where the barrier locations are a function of time (Jacobson and Turco [1995]). Alternatively, a fixed size domain can be chosen that truncates the particle sizes at a given value, which can be selected large enough so that only a small fraction of the overall population is affected. Although the latter approach is simpler to implement, it may result to be more computationally expensive if a wider size domain leads to a larger number of bins.

\footnotetext{
IT The product of the collision and coalescence efficiencies.
} 



\section{SUMMARY}

First principles models of nuclear debris formation have been proposed in the past but were not widely implemented because of the high computational cost (Miller [1960]). Semianalytical models were developed instead, which form the basis of operational codes in use today (Norment [1979]). The evolution of computational power makes it possible now to consider a wider use of first principles approaches and a reduction in the reliance on parameterizations. This is particularly important if predicting capabilities are to be extended beyond the type of scenarios that dominated nuclear tests during the 20th century.

The description presented here offers a first order approximation to this problem, trying to balance a first principles approach that is able to resolve features of interest, with moderate computational costs, making it suitable as a research or semioperational tool. The problem of nuclear debris formation is, at the time of writing, an area of active research, and the current priority is understanding the physical processes that play an important role in the development of the observed debris characteristics.

Although there are parallels between the problem considered here and other systems in which particulates form from a gas phase, there are also unique challenges, such as a wide range of particle sizes, a composition that varies not only because of chemistry but also because of radioactive decay, and a large number of species that are present in very different mass fractions.

The models described here are taken from the literature and have been developed with different applications in mind, for example in the study of heat exchangers in industrial settings (Gyarmathy [1982]) or the evolution of aerosols in the atmosphere (Seinfeld and Pandis [1997]). Although these regimes will differ from those in a fireball, we believe the principal physical processes, namely nucleation, growth, and agglomeration, still play a dominant role, although their relative importance is likely to be different. For simple configurations, for example one component systems, these models have been validated by comparison with analytical solutions. In the case of the formation of nuclear debris the complexity of the system makes the validation process much more involved.

An additional challenge faced here is the difficulty in experimentally reproducing the conditions in which nuclear debris is formed. There have been attempts at approximating those conditions at the laboratory scale (Dai et al. [2013], Koroglu et al. [2017]), but some parameters, such as the rate of cooling, still do not match those considered to be characteristic of nuclear fireballs. The use of historic data in the form of fallout patterns is also of limited value for validation purposes because the required atmospheric transport models introduce additional uncertainties (Stein et al. [2015]). A better source of data are historic debris samples, which were analyzed at the time of the tests (e.g., Nathans [1969]), and more recently using modern analytical techniques (Eppich et al. [2014]). Nevertheless, the number of samples studied thus far is small, making it difficult to derive conclusions of statistical significance. Apart from these limitations, all these sources of data, likely in aggregate, will play an important role in the validation and future development of these models. 



\section{REFERENCES}

C. E. Adams, N. H. Farlow, and W. R. Schell. The compositions, structures and origins of radioactive fall-out particles. Geochimica et Cosmochimica Acta, 18:42-56, 1960.

P. W. Atkins. Physical chemistry. Oxford University Press, 1986.

F. Butuirat and M. Kielkiewickz. On additivity of coagulation kernels. Ann. Nucl. Energy, 23:1091-1096, 1996.

W. S. Cassata, S. G. Prussin, K. B. Knight, I. D. Hutcheon, B. H. Isselhardt, and P. R. Renne. When the dust settles: stable xenon isotope constraints on the formation of nuclear fallout. J. Environ. Radioact., 137:88-95, 2014.

S. Chandrasekhar. Stochastic problems in physics and astronomy. Rev. Mod. Physics, 15:1-89, 1943.

Z. R. Dai, J. C. Crowhurst, C. D. Grant, K. B. Knight, V. Tang, A. A. Chernov, E. G. Cook, J. P. Lotscher, and I. D. Hutcheon. Exploring high temperature phenomena related to post-detonation using an electric arc. J. Appl. Phys., 114:204901, 2013.

M. H. Davies and J. D. Sartor. Theoretical collision efficiencies for small cloud droplets in Stokes flow. Nature, 215:1371-1372, 1967.

Z. Dodin and T. Elperin. On the collision rate of particles in turbulent flow with gravity. Phys. Fluids, 14: 2921-2924, 2002.

S. Elghobashi and G. C. Truesdell. On the two-way interaction between homogeneous turbulence and dispersed solid particles. I: Turbulence modification. Phys. Fluids A, 5:1790-1801, 1993.

G. R. Eppich, K. B. Knight, T. W. Jacomb-Hood, G. D. Spriggs, and I. D. Hutcheon. Constraints on fallout melt glass formation from a near-surface nuclear test. J. Radioanal. Nucl. Chem., 302:593-609, 2014.

A. J. Fahey, C. J. Zeissler, D. E. Newbury, J. Davis, and R. M. Lindstrom. Postdetonation nuclear debris for attribution. Proc. Natl. Acad. Sci., 107:20207-20212, 2010.

J. Feder, K. C. Russell, J. Lothe, and G. M. Pound. Homogeneous nucleation and growth of droplets in vapours. Adv. Phys., 15:111-178, 1966.

A. Ferrante and S. Elghobashi. On the physical mechanisms of two-way coupling in particle-laden isotropic turbulence. Phys. Fluids, 15:315-329, 2003.

M. S. Finko, D. Curreli, D. G. Weisz, J. C. Crowhurst, T. P. Rose, B. Koroglu, H. B. Radousky, and M. R. Armstrong. A model of early formation of uranium molecular oxides in laser-ablated plasmas. J. Phys. D: Appl. Phys., 50:485201, 2017.

E. C. Freiling. Fractionation III. Estimation of degree of fractionation and radionuclide partition for nuclear debris. Report USNRDL-TR-680, US Naval Radiological Defense Laboratory, 1963.

H. L. Frisch and F. C. Collins. Diffusional processes in the growth of aerosol particles. J. Chem. Phys., 20: 1797-1803, 1952. 
H. L. Frisch and F. C. Collins. Diffusional processes in the growth of aerosol particles. II. J. Chem. Phys., 21:2158-2165, 1953.

N. A. Fuchs. Evaporation and droplet growth in gaseous media. Pergamon, 1959.

F. Gelbard and J. H. Seinfeld. The general dynamic equation for aerosols. J. Colloid Interface Sci., 68: 363-382, 1979.

F. Gelbard and J. H. Seinfeld. Simulation of multicomponent aerosol dynamics. J. Colloid Interface Sci., 78:485-501, 1980.

F. Gelbard, Y. Tambour, and J. H. Seinfeld. Sectional representations for simulating aerosol dynamics. $J$. Colloid Interface Sci., 76:541-556, 1980.

F. Gelbard, J. W. Fitzgerald, and W. A. Hoppel. A one-dimensional sectional model to simulate multicomponent aerosol dynamics in the marine boundary layer. 3. Numerical methods and comparisons to exact solutions. J. Geophys. Res., 103:119-132, 1998.

S. L. Girshick and C. P. Chiu. Kinetic nucleation theory: A new expression for the rate of homogeneous nucleation from an ideal supersaturated vapor. J. Chem. Phys., 93:1273-1277, 1990.

G. Giuli, G. Pratesi, S. G. Eeckhout, C. Koeberl, and E. Paris. Iron reduction in silicate glass produced during the 1945 nuclear test at the Trinity site (Alamogordo, New Mexico, USA). Geol. Soc. Am. Spec. Publ., 465:653-660, 2010.

G. Gyarmathy. The spherical droplet in gaseous carrier streams: Review and synthesis. Multiphase Sci. Tech., 1:99-279, 1982.

M. Z. Jacobson and R. P. Turco. Simulating condensational growth, evaportation, and coagulation of aerosols using a combined moving and stationary grid size. Aerosol Sci. Technol., 22:73-92, 1995.

M. Z. Jacobson, R. P. Turco, E. J. Jensen, and O. B. Toon. Modeling coagulation among particles of different composition and size. Atmos. Environ., 28:1327-1338, 1994.

Lord Kelvin. On the equilibrium of vapour at a curved surface of a liquid. Proc. Roy. Soc. Edinb., 7:63-68, 1870.

B. Koroglu, M. Mehl, M. R. Armstrong, J. C. Crowhurst, D. G. Weisz, J. M. Zaug, Z. Dai, H. B. Radousky, A. Chernov, E. Ramon, E. Stavrou, K. Knight, A. L. Fabris, M. A. Cappelli, and T. P. Rose. Plasma flow reactor for steady state monitoring of physical and chemical processes at high temperatures. Rev. Sci. Instrum., 88:093506, 2017.

B. Koroglu, S. Wagnon, Z. Dai, J. C. Crowhurst, M. R. Armstrong, D. Weisz, M. Mehl, J. M. Zaug amd H. B. Radousky, and T. P. Rose. Gas phase chemical evolution of uranium, aluminium, and iron oxides. Sci. Rep., 8:10451, 2018.

J. L. Magee. Mechanisms of fractionation. Office Memorandum M-7140, Los Alamos National Laboratory, 1953.

M. R. Maxey. The gravitational settling of aerosol particles in homogeneous turbulence and random flow fields. J. Fluid Mech., 174:441-465, 1987. 
C. F. Miller. A theory of formation of fallout from land-surface nuclear detonations and decay of the fission products. Report USNRDL-TR-425, Naval Radiological Defense Laboratory, 1960.

M. W. Nathans. The specific activity of nuclear debris from ground surface bursts as a function of particle size. Advan. Chem. Ser., 93:352-359, 1969.

M. W. Nathans, R. Thews, and I. J. Russell. The particle size distribution of nuclear cloud samples. Advan. Chem. Ser., 93:360-380, 1969.

H. G. Norment. DELFIC. Department of Defense fallout prediction system. Volume I — Fundamentals. Report DNA 5159F-1, Defense Nuclear Agency, 1979.

P. Peeters, C. C. M. Luijten, and M. E. H. van Dongen. Transitional droplet growth and diffusion coefficients. Int. J. Heat Mass Transfer, 44:181-193, 2001.

A. Prakash, A. P. Bapat, and M. R. Zachariah. A simple numerical algorithm and software for solution of nucleation, surface growth, and coagulation problems. Aerosol Sci. Technol., 37:892-898, 2003.

G. Saffman and J. S. Turner. On the collision of drops in turbulent clouds. J. Fluid Mech., 1:16-30, 1956.

J. H. Seinfeld and S. N. Pandis. Atmospheric chemistry and physics: From air pollution to climate change. Wiley, 1997.

M. Smoluchowski. Experiments on a mathematical theory of kinetic coagulation of colloid solutions. Z. Physik. Chem., 92:129-168, 1917.

A. F. Stein, R. R. Draxler, G. D. Rolph, B. J. B. Stunder, M. D. Cohen, and F. Ngan. NOAA's HYSPLIT atmospheric transport and dispersion modeling system. Bull. Am. Meteorol. Soc., 96:2059-2077, 2015.

T. H. Tsang and A. Rao. Comparison of different numerical schemes for condensational growth of aerosols. Aerosol Sci. Technol., 9:271-277, 1988.

M. Volmer and A. Weber. Keimbildung in übersättigten gebilden. Z. Phys. Chem., 119:277-301, 1926.

L. P. Wang, A. S. Wexler, and Y. Zhou. Statistical mechanical descriptions of turbulent coagulation. Phys. Fluids, 10:2647-2651, 1998.

L. P. Wang, O. Ayala, S. E. Kasprzak, and W. W. Grabowski. Theoretical formulation of collision rate and collision efficiency of hydrodynamically interacting cloud droplets in turbulent atmosphere. J. Atmos. Sci., 62:2433-2450, 2005.

P. G. Wright. On the discontinuity involved in diffusion across an interface (the $\Delta$ of Fuchs). Discuss. Farad. Soc., 30:100-112, 1960.

H. Zhang, G. Sharma, S. Dhawan, D. Dhanraj, Z. Li, and P. Biswas. Comparison of discrete, discrete-sectional, modal and moment models for aerosol dynamics simulations. Aerosol Sci. Technol., 54:739-760, 2020. 
